\title{
Controlling an upper-limb exoskeleton by EMG signal while carrying unknown load
}

\author{
Benjamin Treussart ${ }^{1}$, Franck Geffard ${ }^{1}$, Nicolas Vignais ${ }^{2}$ and Frederic Marin ${ }^{3}$
}

\begin{abstract}
Implementing an intuitive control law for an upper-limb exoskeleton dedicated to force augmentation is a challenging issue in the field of human-robot collaboration. The aim of this study is to design an innovative approach to assist carrying an unknown load. The method is based on user's intentions estimated through a wireless EMG armband allowing movement direction and intensity estimation along 1 Degree of Freedom. This control law aimed to behave like a gravity compensation except that the mass of the load does not need to be known. The proposed approach was tested by 10 participants on a lifting task with a single Degree of Freedom upper-limb exoskeleton. Participants performed it in three different conditions : without assistance, with an exact gravity compensation and with the proposed method based on EMG armband (Myo Armband). The evaluation of the efficiency of the assistance was based on EMG signals captured on seven muscles (objective indicator) and a questionnaire (subjective indicator). Results showed a statically significant reduction of mean activity of the biceps, erector spinae and deltoid by $20 \% \pm 14,18 \% \pm 12$ and $25 \% \pm 16$ respectively while comparing the proposed method with no assistance. In addition, similar muscle activities were found both in the proposed method and the traditional gravity compensation. Subjective evaluation showed better precision, efficiency and responsiveness of the proposed method compared to the traditional one.
\end{abstract}

\section{INTRODUCTION}

Industrial workers are performing repetitive physical tasks, which expose them to musculoskeletal disorders (MSD) [1]. MSD is a major public health issue, with an impact on workers' integrity and economics. Indeed, MSD are causing both loss in productivity and high healthcare costs. According to [2], MSD have led to the loss of 10 millions work days in France in 2012, with an average cost of $21 \mathrm{k} €$ per case of MSD. A way to prevent MSDs could be to assist the workers during forceful exertions, e.g. load carrying tasks, thus relieving the strain they endure.

In this context, exoskeletons could become a promising solution for industrial load carrying. Although exoskeletons can be backdrivable (see sec. III-A), helping manipulation of known loads [3], the way they can assist in carrying various and unknown loads in a efficient manner is yet to be found. The knowledge of the user's intention could help in this matter. Surface electromyography (EMG) signal processing

\footnotetext{
${ }^{1}$ Benjamin Treussart and Franck Geffard are with LASR, SRI, CEA-List, Saclay, France benjamin.treussart@cea.fr franck.geffardecea.fr

${ }^{2}$ Nicolas Vignais is with CIAMS, Univ. Paris-sud, Univ. Paris Saclay, Orsay, France and CIAMS, Univ. d' Orleans, Orleans, France nicolas.vignais@u-psud.fr

${ }^{3}$ Frederic Marin is with Biomecanique et Bioingenierie UMR CNRS 7338, Universite de Technologie de Compiegne, Compiegne, France ferderic.marineutc.fr
}

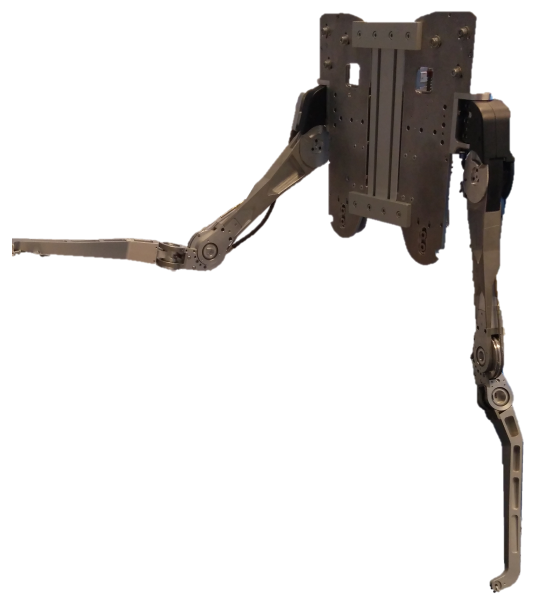

Fig. 1: Upper-limb exoskeleton

demonstrated reliability in estimating the muscle force [4], and, consequently, it may be a relevant bio-signal to capture the motion intention.

Given a context of industry-oriented application, a control scheme based on an EMG armband was designed and evaluated in the present study. This control is initialized with a specific calibration procedure described in IV-A.

\section{RELATED WORKS}

\section{A. Human-Robot Interaction}

One of the most common type of interaction with a robotic device is an interaction of a serial-type. The robot acts as a prolongation of the user (prosthesis [5], teleoperation). But, recently, the design of active exoskeletons and collaborative robots (which the user would manipulate an object in coordination [6] with) have introduced new paradigm of interaction where the robot acts in parallel with the human. In this case, the action of the robot not only supports a larger part of the load, but also interacts with the human who is carrying a fraction of this load. This is the situation considered in this study.

In this context, two main approaches may be proposed to perform the task in an intuitive way, i.e. that does not require training and/or an important change of behavior. The first one is to assume prior knowledge of the task to program the robot accordingly (like with virtual guides [7] or gravity compensation (CG) [8]). The second approach is more related to a typical professional environment, in which the operator does not know the weight of the load she/he is 
going to carry. In that case the intention of the user can be used to control the robot.

In this study, the targeted behavior for the proposed method is the exact payload gravity compensation [8] under the constraint of not knowing the mass of the load. This type of control enables the user to rest their muscles before the point of interaction. Thus, if an exoskeleton is attached around the forearm or wrist, then the muscles involved before this segment, i.e. the biceps or triceps, can be relieved.

\section{B. Muscle activity for intention detection}

EMG signals have already been used to control robotic devices [9], [10]. This biocybernetic approach uses EMG signals into two different manners: discretely or continuously. The discrete-EMG method uses pattern recognition based on handcrafted features and a classifier trained with them [11] or some end-to-end neural networks [12]. The main inconvenience of this method of intention detection is its lack of flexibility. Indeed, there is a trade-off between the panel of actions on one hand and the training required and reliability of the classification, which depends on the number of classes, on the other hand. In the case of a directionnal control, one class may correspond to one direction with an imposed speed [10].

On the other hand, a continuous-EMG method seems more adequate. However, it also presents disadvantages. First of all, it is more complex, due to the fact that the relations between the features of the EMG signals and the movement are highly non-linear [13]. In [14], a continuous $3 \mathrm{D}$ estimation of the position of the hand is performed with the use of 9 electrodes targeting specific muscles. Such precise requirements in the placement of the electrodes are laborious and examiner-dependent. Although these methods are adapted to teleoperation or prosthesis assistance, using a continuous-EMG method to control an exoskeleton while carrying unknown load appears difficult. In that latter situation, the assistance has to be provided even if the muscle activity decreases, which can not be considered with the continuous-EMG method. In the current study, a compromise between discrete and continuous approaches has been developed to evaluate the user's intentions. A low-cost surface EMG device has been used to foresee a practical use-case. To summarize, the aim of this study is to assist a user carrying a load without prior knowledge on the load's mass, and based on the user's muscle activity.

\section{System SPECIFICATIONS}

\section{A. Exoskeleton}

The exoskeleton used in this study is an under-actuated upper-body backdrivable type (figure 1). Each side consists of two segments (upper-arm and forearm) and four joints. Two of the joints are passive $\left(\theta_{1}\right.$ and $\left.\theta_{4}\right)$ and the other two are proportionally linked and powered by the same actuator $\left(\theta_{3}=1.5 * \theta_{2}\right)$ [15]. The end effector of the exoskeleton, that pulls the hand upward, is attached to the palm of the user's hand by a strap. "Backdrivable" behavior is "the nature that when the force is added to the output axis, the output axis

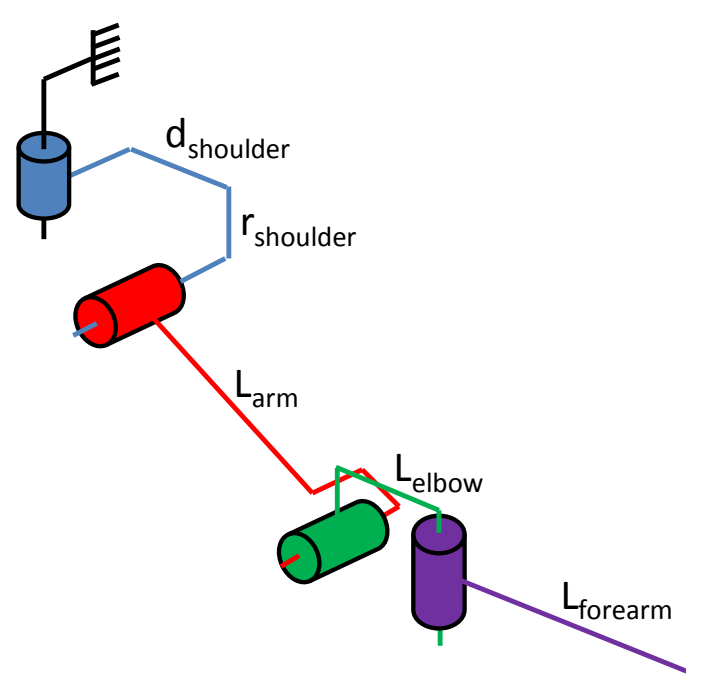

(a) Representation of the DH parameters

\begin{tabular}{|c|c|c|c|c|}
\hline & $\alpha_{i}$ & $d_{i}$ & $r_{i}$ & $\theta_{i}$ \\
\hline Frame 1 & 0 & 0 & 0 & $\theta_{1}$ \\
Frame 2 & $+\frac{\pi}{2}$ & $d_{\text {shoulder }}$ & $r_{\text {shoulder }}$ & $\theta_{2}$ \\
Frame 3 & 0 & $L_{\text {arm }}$ & 0 & $\theta_{3}=1.5 * \theta_{2}$ \\
Frame 4 & $-\frac{\pi}{2}$ & $L_{\text {elbow }}$ & 0 & $\theta_{4}$ \\
Frame 5 & 0 & $L_{\text {forearm }}$ & 0 & 0 \\
\hline
\end{tabular}

(b) Table of the DH parameters

Fig. 2: Geometry of the BHV2 exoskeleton

is moved by this force and this motion is conveyed to the input axis and the input axis is moved by this motion in the case of actuators or power transmitting mechanism" [16].

During the interaction, human and robotic forearm dynamics are coupled, which can be modeled as follows [17]:

$$
\left\{\begin{array}{l}
J_{r} \ddot{\Theta}_{r}+b_{r} \dot{\Theta}_{r}+F_{N L}+Q_{r}\left(\Theta_{r}\right)=\tau_{r}-\tau_{i} \\
J_{h} \ddot{\Theta}_{h}+J_{h}^{t} F_{e}+b_{h} \dot{\Theta}_{h}+Q_{h}\left(\Theta_{h}\right)=\tau_{h}+\tau_{i}
\end{array}\right.
$$

Where $\tau_{i}$ is the interaction torque between human and robot limbs, and $J$ - the moment of inertia, $b$ - the viscosity, $F_{N L}$ - the non-linear friction and $Q$ the gravity torque. The subscripts $r$ and $h$ denote similar quantities related to robot and human systems, respectively. We are considering a quasi static interaction and we compensate the effect of gravity on the robot (Backdrivability ensures that $F_{N L}$ is low [3]), which give us:

$$
\tau_{h}=-\tau_{r}+J_{h}^{t} F_{e}+Q_{h}\left(\Theta_{h}\right)-F_{N L}
$$

\section{B. EMG Measure}

The overall intention detection solution is expected to be functional, easy to deploy, low-cost, operational in realtime. The Myo-Armband (Thalmic Labs, Ontario) meets all specifications. The armband was positioned around the arm to capture biceps and triceps muscle activities, rather 
than on the foreman as it was originally designed. Indeed, because the exoskeleton is tied to the palm of the user's hand, the muscle of the forearm will not be relieved by the assistance of the exoskeleton, unlike the biceps and triceps. The Armband is composed of eight pairs of dry electrodes. We optimized the longitudinal placement to maximize the EMG signal empirically. The raw output of the armband is a zero-mean signal coded over 8 bit, it has no unit and is comprised between -126 and +127 .

\section{Control SCHEME}

\section{A. Intention Estimation}



Fig. 3: Estimation of the user's intention (Intensity ${ }_{B}$ and Intensity $_{T}$ correspond respectively to the intensity of the Biceps activation and Triceps activation)

The objective of the present study is to assist load carrying by reproducing behavior similar to a gravity compensation. Our contribution is to do so without prior knowledge of the object's mass by exploiting user's intentions. Intentions are identified with the procedure presented on figure 31 . More precisely, a short calibration step with a 2-minute EMG recording allowed the definition of the four classes of action, and the definition of intensity parameters of the model (equation 3) [18]. The first step to estimate the intensity of the movement intended is to sum and rectify the signal from the 8 sensors of the armband, then to filter the result with a Butterworth filter (order 2 and a $2 \mathrm{~Hz}$ cutoff frequency) and finally to convert filtered value to a torque value using the calibrated model. And simultaneously, the direction is estimated using a convolutional neural network.

$$
\tau=u^{a} * e^{(c-b * u)}
$$

$\tau$ being the modeled torque, $u$ the EMG signal and $a, b$ and $c$ the parameters to be determined for the biceps and triceps from calibration.

Thus, an objective value of the user's intentions as a torque is determined. This approach allows tuning a control scheme independently from the user's EMG specificity. In this context the intention of the user is considered to be a signed value of torque.

\footnotetext{
${ }^{1}$ The design and evaluation of the module of intention detection and its calibration is currently under review
}

\section{B. Control}

The main motivation to design a type of control law close to a gravity compensation, rather than some type of proportional control [19], [20] is to allow a full support by the exoskeleton without specific muscle activity of the operator.

A schematic representation of the control loop was represented in figure 4 showing a torque control loop where the muscle relaxation objective was represented by the human elbow torque setpoint $\tau_{d e s}$ that was constantly set to 0 . $\tau_{\text {cons }}$ was the control torque applied to the exoskeleton (ideally the torque really provided by the exoskeleton). $F_{\text {inter }}$ corresponded to part of the interaction wrench between the exoskeleton and the operator. $V_{\text {muscles }}$ corresponded to the muscular myoelectric signal that could be measured by the Myo ArmBrand. The system to be controlled was the exoskeleton/operator pair in which the weight of the load was carried by the operator. The integral corrector is widely used in torque control, allowing both the cancellation of gravity and friction, as well as the reduction of inertia [21], [22]. In the present study, this integral corrector was used to control the torque, not of the robot, but of the operator's elbow through the exoskeleton.

Although often present in torque control, the dead zone block is generally not represented. Nevertheless, in the present study, this dead zone block was represented because it provided a crucial adjustment parameter, allowing a more efficient use of EMGs with integral corrector (later called DZ-I corrector for Dead Zone Integral corrector). In addition to the characteristics mentioned above, the DZ-I corrector also has the advantage of filtering errors in the intention detection system. This is of crucial interest in our case, since the direction estimated by the module described in figure 3 is less reliable at low intensity. The dead zone first allows to cancel the impact of such errors. In addition, even isolated errors at higher intensities would be absorbed by the fact that an integrator was used. This usually needs to be solved by a harsh low-pass filtering in the case of the purely proportional approach, introducing phase lag and thus low bandwidth.

The DZ-I corrector is particularly interesting when handling loads with a backdrivable robotic device. Indeed, once the weight of the load is compensated, since the frictions forces are very small by design, it only leaves the inertial forces. So, if the threshold of the dead zone is set to a high enough value, the load can be moved without changing the assisting torque, thus yielding a behavior similar to a $\mathrm{CG}$ algorithm.

In figure 4 , there are two parameters to tune: $K$ for the integrator and threshold, for the dead zone. Also, the integration was calculated with (intensity - threshold) rather than intensity alone in order to have a more progressive evolution of the assisting torque. threshold is the second parameter to tune, it influences the limite-cycle oscillation around null torque but also the potential static error.

The proposed control was tuned for and by an expert, i.e. someone who is trained on this approach, and the resulting 


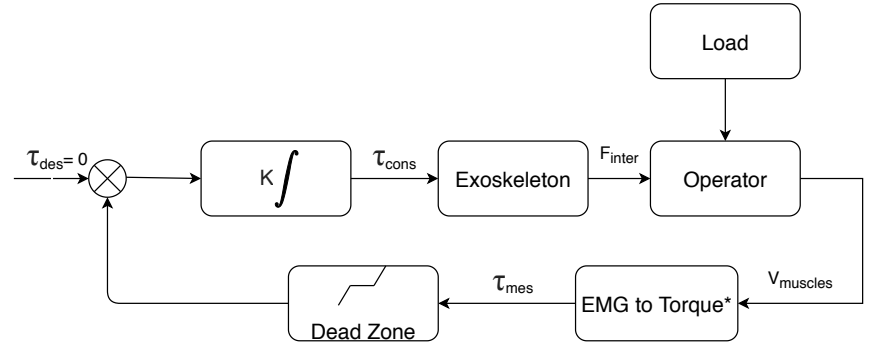

Fig. 4: Control scheme of the proposed method.

*block containing figure 3

parameters were used for all the participants in section V.

\section{EXPERIMENTAL PROTOCOL}

To evaluate the proposed control law, an experimental protocol has been conducted. 10 subjects participated to this study ( 8 men and 2 women, aged $27.8 \pm 11.6,176 \pm 8 \mathrm{~cm}$ and $78 \pm 17 \mathrm{~kg}$ ). All participants gave their informed consent to take part to this study. The participants had to lift and put down a load of $5 \mathrm{~kg}$ repeatedly at natural speed and in three different situations : without assistance (No-Exo), with our proposed control (DZ-I), and with gravity compensation (CG).

The participants were equipped with seven EMG sensors (DataLite, Biometrics Ltd, Newport, UK) targeting muscles relevant for biomechanical analysis during load carrying: biceps, triceps, deltoid, trapezius, erector spinae, soleus, tibialis anterior. The sensors were placed only on the left side, as the movement was performed in the sagittal plane. Moreover, the right arm was already equipped with the Myo Armband. The goal of these measurements was to have an objective evaluation of the muscle activity during different situations. The EMG sensors were placed according to the SENIAM recommandations [23], and the sample frequency was fixed to $1000 \mathrm{~Hz}$.

Participants were also asked to fill up a questionnaire for subjective analysis [24]. This questionnaire assessed how the user perceived the different situations and if the perception was in accordance with the objective evaluation.

One move set of the task was to lift a load up to a high mark, bring it down to a middle mark, high up again and finally put the load down (figure 5). For each situation the participants had to perform 6 repetitions of this move sets. Before starting the recordings the participants were allowed to make at most two complete movements in order for them to have an idea of how the different control laws would react.

As mentioned in section IV-B, the proposed control law was tuned with two parameters. The gain was set to get the fastest response time without causing stability issues and the threshold high enough to enable low dynamics up and down movements without triggering a change in the assisting torque.

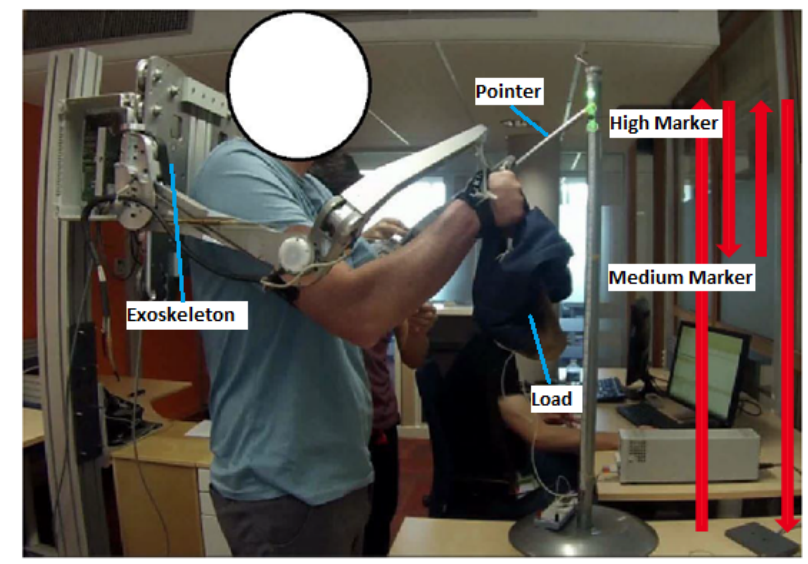

Fig. 5: Experimental set up

\section{RESUlts}

To give an overview of the results, figure 6 shows the recordings of one participant's move set for each situation. Only EMG signals of the triceps and biceps have been displayed for reading purposes.

\section{A. Control Performance}

The torque of reference was considered as the torque that compensates gravity when the user is lifting the object. On figure 7 , the average RMS error per move set for each participant, and the average difference between the torque output and the torque of reference were presented. The second value was calculated in order to observe if the proposed control approach had a tendency to undershoot or overshoot. The values were calculated between the moment when the object was lifted up and the moment it was put down. It can be seen that, except for the first participant, the RMS error is $0.038 \pm 0.012 N . m$ on average, which is equivalent to $6.99 \pm 2.27 \mathrm{~N}$ at the hand of the users. The first participant has been identified as an outlier following the quartile analysis. It could be explained by the fact that the participant had a stiff and tensed behavior that made it difficult for the proposed method to operate. Such an issue could be solved by according a proper training time to the users.

By examining the average differences, it was observed that the proposed control did not systematically overshoot or undershoot in the same way for each participant. Based on these results, the existence of a user-specific parameters can be suggested. This parameter would influence the gain of the integrator and could be determined from the participant's reaction time. It may also correspond to interindividual differences when discovering a new paradigm of assistance. These differences might be investigated by designing an experiment with a high number of repetitions in order to analyze the participants' adaptation.

\section{B. Muscle Activity}

The situations without assistance and with gravity compensation assistance were performed by the participants in 


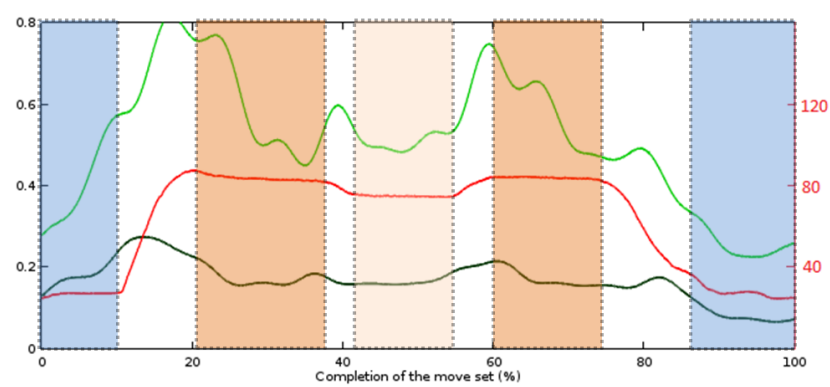

(a) No Exo

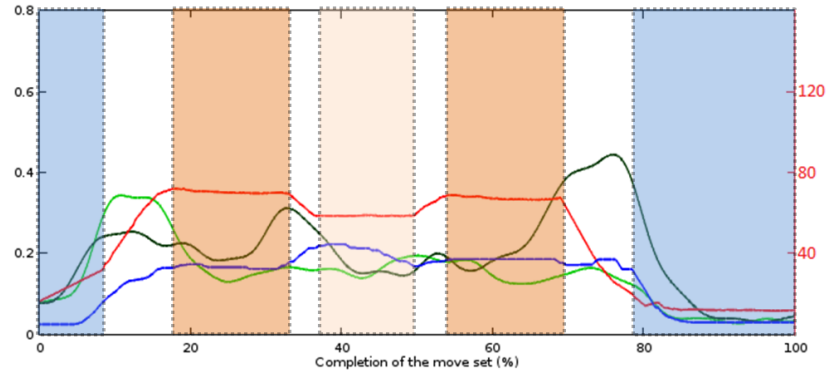

(b) DZI

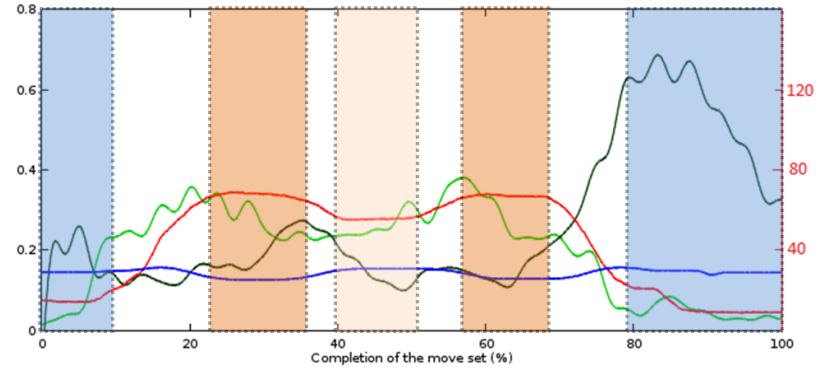

(c) $\mathrm{CG}$

Fig. 6: Recordings of one move set in each situation for one participant. In red, the elbow angle is displayed (right ordinate axis, degrees), in blue the assisting torque (left ordinate axis, N.m) and the two green curves are the level of activation of the biceps and triceps (light and dark respectively, left ordinate axis, normalized with the RMC (see section VI-B)). The different key moments of a move set are highlighted on the graphs : in blue the load is released, in orange the load is lifted up to the high mark and in light orange the load is at the medium mark.

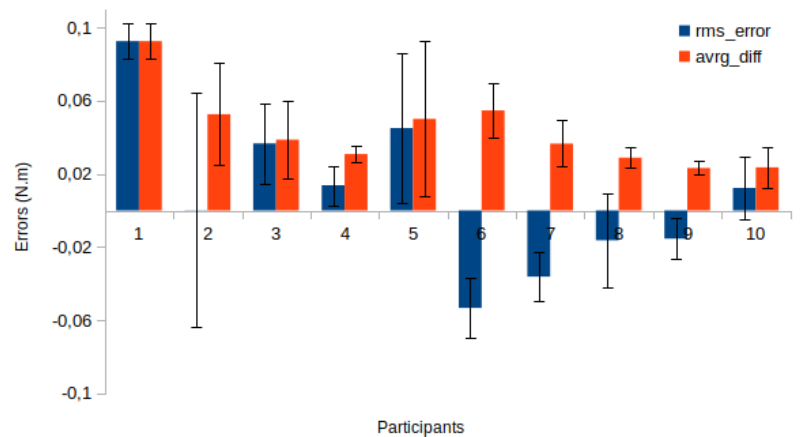

Fig. 7: Precision of the proposed method compared to gravity compensation

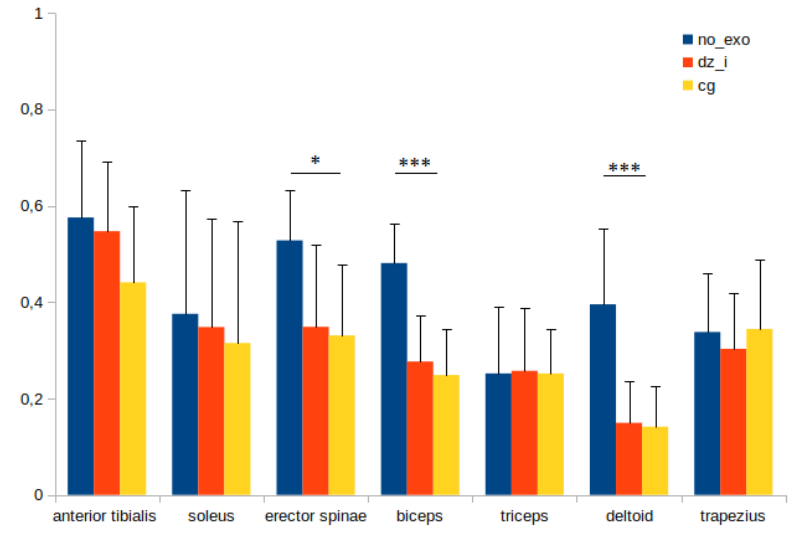

Fig. 8: Comparison of muscle activation between the three conditions $(* p<0.05, * * * p<0.001)$

order to provide baselines to evaluate the performances of the proposed control law regarding the physical relief brought to the user. The processing of the raw EMG sensors followed the recommandations of [25] and [26], a band-pass filter (bidirectionnal $7^{\text {th }}$ order Butterworth $[30 \mathrm{~Hz}, 200 \mathrm{~Hz}]$ ) was first applied, then centered and rectified and finally low-passed. The signal was then normalized with the maximum value measured over all the situations and move sets for every respective muscle and participant (RMC, relative maximum contraction). The signal was parsed and down-sampled to calculate the average muscle activation during each move set. On figure 8, the average activation of each muscle is displayed. While comparing the proposed method with no assistance, results showed a statically significant reduction of mean activity of the biceps, erector spinae and deltoid by $20 \pm 14 \%, 18 \pm 12 \%$ and $25 \pm 16 \%$ respectively $(p<0.001$, $p<0.05$, and $p<0.001$ respectively). Previous studies from the literature reported a decrease in muscle activation of the biceps brachii superior to $20 \%$ when using an active upperlimb exoskeleton [27]-[30], thus reducing the physical load on the operator's arm during manual tasks [31]. Concerning erector spinae muscle, a reduction between 10 to $25 \%$ have been showed during asymmetric lifting tasks with an exoskeleton [32]-[34], confirming the capacity of these devices to prevent back injuries. Although the highest decrease in muscle activation was obtained from the deltoid muscle, comparable values were obtained in previous studies [29]. Similar muscle activities were found both in the proposed method and the traditional gravity compensation for the other muscles.

One of the advantages of the proposed method compared to the gravity compensation is that the assistance torque is based on the effort made by the participant. Thanks to that, the torque is reduced when the object is released, unlike with a regular gravity compensation (behavior similar to passive exoskeleton) where the torque is maintained. To evaluate this difference, the maximum activations during the last part of the movement between the two types of assistance are compared in table 9. For half of the participants there is a 


\begin{tabular}{|c|c|c|c|c|c|c|c|c|c|c|}
\hline Participant & 1 & 2 & 3 & 4 & 5 & 6 & 7 & 8 & 9 & 10 \\
\hline DZI (\% of RMC) & 61 & 23 & 49 & 56 & 31 & 25 & 18 & 28 & 59 & 47 \\
\hline CG (\% of RMC) & 20 & 24 & 85 & 48 & 36 & 73 & 69 & 73 & 53 & 77 \\
\hline Difference & -41 & 01 & 37 & -08 & 05 & 48 & 51 & 45 & -07 & 30 \\
\hline
\end{tabular}

Fig. 9: Maximum activation of the triceps during the last part of the move set

notable decrease in the maximum contraction of the triceps while releasing the object. Excluding the first participant, considered as an outlier in section VI-A, the average difference obtained was $22 \pm 24 \%$ of RMC. After close observation of each move set it was noticed that the participants did not always released the object at the end of the move set. Despite this fact, it could still be noticed that our proposed method tends to put less strain on the triceps compared to the compensation of gravity.

\section{Questionnaire}

The participants answered questions after performing the tasks. The questionnaire was divided in three parts. The first one was about general perception. The second one was related to the comparison of the situation without assistance and the situation with the DZ-I control. And the last one was related to the comparison of the two type of control (DZ-I and CG) (Figure 10). The form used a Likert scale.

The general feedback from the participants was that, even though the CG control was easy to anticipate and required less concentratrion because of its consistency, they felt more efficient with the DZ-I control. This was highlighted by comparing the feeling of control with each method on figure $10 \mathrm{a}$.

Nine participants felt that the proposed method successfully reduced the efforts made by their arms compared to the situation without assistance, and seven felt a relief in their shoulders. When comparing both algorithms (CG and DZ-I), participants felt mostly no difference for some of the inquired areas (cervical area, back, lower limbs). Seven participants felt a greater relief in their arms when comparing our proposed method to the gravity compensation, and five a greater relief in the shoulders (Figure 10b). It is worth noting that one participant felt more strain in the back.

\section{CONCLUSIONS AND FUTURE WORK}

In the present study, a method to assist a user while carrying a load was designed and evaluated. It was based on the EMG signals measured on the arm with a low cost EMG recording system. An experimental protocol was conducted with the help of 10 participants in order to evaluate the proposed method and compare it to a situation without assistance and to a gravity compensation assistance. It was observed that the proposed method was able to approach the ideal torque with an error of $0.038 \pm 0.012 \mathrm{~N} . \mathrm{m}$ on average, which is equivalent to $6.99 \pm 2.27 N$ at the end effector. Thus, the DZ-I control may be used to assist load carrying during industrial manual tasks. It remains to be seen if the torque assistance is accurate enough, considering that



(a)

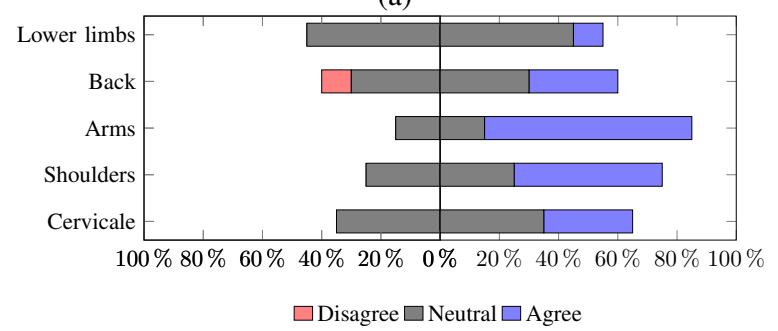

(b)

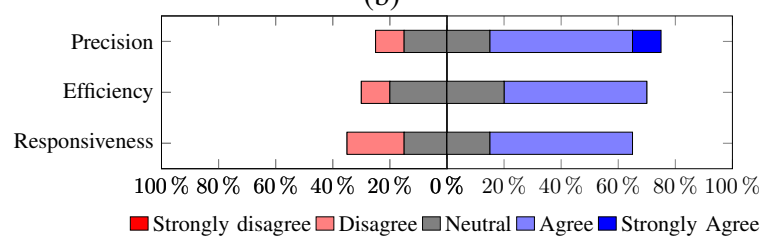

(c)

Fig. 10: (a) Feeling of control with the two methods of assistance

(b) Compared muscle strain with DZI relatively CG

(c) Compared impressions about performances of the $\mathrm{CG}$ and DZI : Precision $=$ Feeling of a better accuracy during the move sets, Efficiency = Feeling of a suitable assistance, Speed $=$ Feeling of a better reaction. The answers were given considering DZI relatively to CG.

this new method bypasses the need of information about the weight of the load (unlike the gravity compensation).

It was also shown that the assistance was effectively reducing the physical strain on the participant (erector spinae, biceps, deltoid muscles). Even though the exoskeleton was attached to a support pole and not carried by the user, those results might be transferred to applications with a lowerbody exoskeleton or to a robot attached to a manipulator as in [35]. In addition, the questionnaire enabled to evaluate the perception of the participants.

The proposed control is more suited for tasks that require carrying various heavy loads and often putting them down; compared to a gravity compensation that is particularly suited for handling a given tool for long periods of time.

As mentioned in section VI, the results were collected from non-expert users. The next step is to extend the number of repetitions in order to observe to observe an adaptation of the human behavior and a subsequent improvement of the subjective feeling. In addition, exploring ways to tune the parameters of the control to different users and their expertise could enhance its ease of use. Another important aspect that will be investigated is the calibration. For now the calibration is entirely user specific, but for deployment purposes it would be interesting to exploit the data from previous users in order to reduce the time spent on calibration. 


\section{REFERENCES}

[1] N. Vignais, M. Miezal, G. Bleser, K. Mura, D. Gorecky, and F. Marin, "Innovative system for real-time ergonomic feedback in industrial manufacturing," Applied ergonomics, vol. 44, no. 4, pp. 566-574, 2013.

[2] INRS. (2015) Troubles musculo-squelettiques - statistiques. [Online]. Available: http://www.inrs.fr/risques/tms-troublesmusculosquelettiques/statistiques.html

[3] P. Garrec, "Design of an anthropomorphic upper limb exoskeleton actuated by ball-screws and cables," Bulletin of the Academy of Sciences of the Ussr-Physical Series, vol. 72, no. 2, p. 23, 2010.

[4] M. Al Harrach, V. Carriou, S. Boudaoud, J. Laforet, and F. Marin, "Analysis of the semg/force relationship using hd-semg technique and data fusion: A simulation study," Computers in biology and medicine, vol. 83, pp. 34-47, 2017.

[5] K. Xu, W. Guo, L. Hua, X. Sheng, and X. Zhu, "A prosthetic arm based on emg pattern recognition," in 2016 IEEE International Conference on Robotics and Biomimetics (ROBIO). IEEE, 2016, pp. 1179-1184.

[6] J. DelPreto and D. Rus, "Sharing the load: Human-robot team lifting using muscle activity," in 2019 International Conference on Robotics and Automation (ICRA). IEEE, 2019, pp. 7906-7912.

[7] S. S. Restrepo, G. Raiola, P. Chevalier, X. Lamy, and D. Sidobre, "Iterative virtual guides programming for human-robot comanipulation," in 2017 IEEE International Conference on Advanced Intelligent Mechatronics (AIM). IEEE, 2017, pp. 219-226.

[8] N. Sylla, V. Bonnet, F. Colledani, and P. Fraisse, "Ergonomic contribution of able exoskeleton in automotive industry," International Journal of Industrial Ergonomics, vol. 44, no. 4, pp. 475-481, 2014.

[9] A. Ajoudani, A. M. Zanchettin, S. Ivaldi, A. Albu-Schäffer, K. Kosuge, and O. Khatib, "Progress and prospects of the human-robot collaboration," Autonomous Robots, pp. 1-19, 2018.

[10] C. DaSalla, J. Kim, and Y. Koike, "Robot control using electromyography (emg) signals of the wrist," Applied Bionics and Biomechanics, vol. 2, no. 2, pp. 97-102, 2005

[11] P. Phukpattaranont, S. Thongpanja, K. Anam, A. Al-Jumaily, and C. Limsakul, "Evaluation of feature extraction techniques and classifiers for finger movement recognition using surface electromyography signal," Medical \& biological engineering \& computing, pp. 1-13, 2018.

[12] M. Gandolla, S. Ferrante, G. Ferrigno, D. Baldassini, F. Molteni, E. Guanziroli, M. Cotti Cottini, C. Seneci, and A. Pedrocchi, "Artificial neural network emg classifier for functional hand grasp movements prediction," Journal of International Medical Research, vol. 45, no. 6, pp. 1831-1847, 2017.

[13] F. E. Zajac, "Muscle and tendon properties models scaling and application to biomechanics and motor," Critical reviews in biomedical engineering, vol. 17, no. 4, pp. 359-411, 1989.

[14] P. K. Artemiadis and K. J. Kyriakopoulos, "An emg-based robot control scheme robust to time-varying emg signal features," IEEE Transactions on Information Technology in Biomedicine, vol. 14, no. 3, pp. 582-588, 2010.

[15] P. Garrec, Y. Perrot, D. Ponsort, and A. Riglet, "Patent: Exoskeleton arm having an actuator," 2012, uS9375325B2.

[16] T. Ishida and A. Takanishi, "A robot actuator development with high backdrivability," in Robotics, Automation and Mechatronics, 2006 IEEE Conference on. IEEE, 2006, pp. 1-6.

[17] S. Bastide, N. Vignais, F. Geffard, and B. Berret, "Interacting with a "transparent" upper-limb exoskeleton: A human motor control approach," in 2018 IEEE/RSJ International Conference on Intelligent Robots and Systems (IROS). IEEE, 2018, pp. 4661-4666.

[18] K. Ullah and J.-H. Kim, "A mathematical model for mapping emg signal to joint torque for the human elbow joint using nonlinear regression," in ICARA 2009. IEEE, 2009, pp. 103-108.

[19] T. Lenzi, S. De Rossi, N. Vitiello, and M. C. Carrozza, "Proportional emg control for upper-limb powered exoskeletons," in 2011 Annual International Conference of the IEEE Engineering in Medicine and Biology Society. IEEE, 2011, pp. 628-631.

[20] T. Lenzi, S. M. M. De Rossi, N. Vitiello, and M. C. Carrozza, "Intention-based emg control for powered exoskeletons," IEEE transactions on biomedical engineering, vol. 59, no. 8, pp. 2180-2190, 2012.

[21] F. Geffard, C. Andriot, A. Micaelli, and G. Morel, "On the use of a base force/torque sensor in teleoperation," in Proceedings 2000 ICRA. Millennium Conference. IEEE International Conference on Robotics and Automation. Symposia Proceedings (Cat. No. 00CH37065), vol. 3. IEEE, 2000, pp. 2677-2683.

[22] D. Williams and O. Khatib, "Improved force control for conventional arms using wrist-based torque feedback," in Experimental Robotics IV. Springer, 1997, pp. 516-525.

[23] H. J. Hermens, B. Freriks, R. Merletti, D. Stegeman, J. Blok, G. Rau, C. Disselhorst-Klug, and G. Hägg, "European recommendations for surface electromyography," Roessingh research and development, vol. 8, no. 2, pp. 13-54, 1999.

[24] L. Wioland, L. Debay, and J. Atain-Kouadio, "Acceptation des exosquelettes par les opérateurs: étude," $130 \mathrm{Avril}$, p. 45.

[25] T. S. Buchanan, D. G. Lloyd, K. Manal, and T. F. Besier, "Neuromusculoskeletal modeling: estimation of muscle forces and joint moments and movements from measurements of neural command," Journal of applied biomechanics, vol. 20, no. 4, pp. 367-395, 2004.

[26] A. C. McDonald, K. Sanei, and P. J. Keir, "The effect of high pass filtering and non-linear normalization on the emg-force relationship during sub-maximal finger exertions," Journal of Electromyography and Kinesiology, vol. 23, no. 3, pp. 564-571, 2013.

[27] J. Naito, G. Obinata, A. Nakayama, and K. Hase, "Development of a wearable robot for assisting carpentry workers," International Journal of Advanced Robotic Systems, vol. 4, no. 4, p. 48, 2007.

[28] H. Kobayashi, T. Aida, and T. Hashimoto, "Muscle suit development and factory application," International Journal of Automation Technology, vol. 3, no. 6, pp. 709-715, 2009.

[29] Y. Muramatsu, H. Kobayashi, Y. Sato, H. Jiaou, T. Hashimoto, and H. Kobayashi, "Quantitative performance analysis of exoskeleton augmenting devices-muscle suit-for manual worker," International Journal of Automation Technology, vol. 5, no. 4, pp. 559-567, 2011.

[30] H. Lee, W. Kim, J. Han, and C. Han, "The technical trend of the exoskeleton robot system for human power assistance," International Journal of Precision Engineering and Manufacturing, vol. 13, no. 8, pp. 1491-1497, 2012.

[31] M. P. De Looze, T. Bosch, F. Krause, K. S. Stadler, and L. W. O'Sullivan, "Exoskeletons for industrial application and their potential effects on physical work load," Ergonomics, vol. 59, no. 5, pp. 671681, 2016.

[32] M. Abdoli-E, M. J. Agnew, and J. M. Stevenson, "An on-body personal lift augmentation device (plad) reduces emg amplitude of erector spinae during lifting tasks," Clinical Biomechanics, vol. 21, no. 5, pp. 456-465, 2006.

[33] M. Abdoli-Eramaki, J. M. Stevenson, S. A. Reid, and T. J. Bryant "Mathematical and empirical proof of principle for an on-body personal lift augmentation device (plad)," Journal of biomechanics, vol. 40, no. 8, pp. 1694-1700, 2007.

[34] M. Abdoli-e and J. M. Stevenson, "The effect of on-body lift assistive device on the lumbar $3 \mathrm{~d}$ dynamic moments and emg during asymmetric freestyle lifting," Clinical Biomechanics, vol. 23, no. 3, pp 372-380, 2008.

[35] H. Yu, I. S. Choi, K.-L. Han, J. Y. Choi, G. Chung, and J. Suh, "Development of a upper-limb exoskeleton robot for refractory construction," Control Engineering Practice, vol. 72, pp. 104-113, 2018. 